\title{
Stellar magnetism through the polarized eyes of the FORS1 instrument
}

\author{
S. Bagnulo*, J.D. Landstreet ${ }^{\dagger}$, L. Fossati** and O. Kochukhov ${ }^{\ddagger}$ \\ *Armagh Observatory, College Hill, Armagh BT61 9DG, UK \\ ${ }^{\dagger}$ Physics \& Astronomy Department, The University of Western Ontario, London, Ontario, Canada \\ N6A $3 K 7$. \\ ** Department of Physics and Astronomy, Open University, Walton Hall, Milton Keynes MK7 6AA \\ ${ }^{\ddagger}$ Department of Physics and Astronomy, Uppsala University, 75120 Uppsala, Sweden
}

\begin{abstract}
During the last decade, the FORS1 instrument of the ESO Very Large Telescope has been used to obtain low resolution circular polarized spectra for about 500 stars, with the aim of measuring their mean longitudinal magnetic fields. Magnetic field estimates were obtained by different authors, using different software tools. Several interesting detections were obtained at a $3 \sigma$ level; some of them were eventually confirmed by follow-up investigations, some of them were not. This raises issues about the reliability of the stated uncertainties of some of the published field values. To investigate these problems, we have developed a semi-automatic procedure for magnetic field determination, which includes self-consistent checks for field detection reliability. We have applied our procedure to the full content of single star ("fast mode") circular spectropolarimetric measurements of the FORS1 archive, and explored the details and interagreement of various methods for data reduction. We have finally produced a catalogue of FORS1 longitudinal field measurements which includes about 1000 entries. Here we critically review the previously published FORS1 measurements, and, based on our results, we suggest that the incidence of the magnetic field in various classes of stars should be revised.
\end{abstract}

Keywords: Polarimetry - Photometric, polarimetric, and spectroscopic instrumentation-Magnetic and electric fields; polarization of starlight

PACS: 95.75.Hi - 95.55.Qf - 97.10.Ld

\section{INTRODUCTION}

The FORS1 instrument (Appenzeller et al. 1998) of the ESO VLT has been extensively used for magnetic field measurements in various classes of stars. The large majority of FORS1 measurements have been published in the literature, and we thought that a general catalogue would serve to obtain an overview (even though biased on target selection) of the incidence of the magnetic fields in various kinds of stars. However, a catalogue compiled using published material would suffer from the lack of homogeneity in the way data have been treated. Furthermore, over time, new ideas for data reduction and quality checks have improved the reliability of FORS1 magnetic measurements, which calls for a revision of earlier data. We also noted that the literature of FORS magnetic field measurements includes a certain amount of controversial detections, e.g.: (i) FORS detections not confirmed by observations obtained with other instruments; (ii) FORS detections not confirmed by further FORS measurements; (iii) FORS detections not confirmed by the re-analysis of the same data by other groups; (iv) FORS detections not confirmed by the re-analysis of the same data performed by the same group who 
originally claimed detection.

The release of the FORS pipeline for spectropolarimetric data (Izzo et al. 2010, 2011) gave us the opportunity to develop a reduction method potentially more accurate than what available in the past. Compared to the reduction of raw data coming from individual observing runs, the mass-production of reduced spectra offers a few advantages in terms of quality of the final products, as the results can be checked on a large statistical basis. Therefore we have decided to tackle the task of re-reducing the entire FORS archive of spectropolarimetric data obtained in single target mode ("fast mode"), and compile a new catalogue. In this paper we report on some of our most important findings.

In Sect. 2 and 3 we give details about data reduction and field measurements techniques, and in Sects. 4 and 5 we present our results and conclusions.

\section{MAGNETIC FIELD MEASUREMENTS}

The mean longitudinal magnetic field is obtained from the Stokes parameters $I$ and $V$ using the relationship

$$
\frac{V}{I}=-g_{\mathrm{eff}} C_{Z} \lambda^{2} \frac{1}{I} \frac{\mathrm{d} I}{\mathrm{~d} \lambda}\left\langle B_{z}\right\rangle
$$

where $g_{\text {eff }}$ is the effective landé factor, and

$$
C_{Z}=\frac{e}{4 \pi m_{\mathrm{e}} c^{2}} \quad\left(\simeq 4.67 \times 10^{-13} \mathrm{~A}^{-1} \mathrm{G}^{-1}\right)
$$

where $e$ is the electron charge, $m_{\mathrm{e}}$ the electron mass, $c$ the speed of light. It should be reminded that Eq. (1) is subject to several important limitations: (i) it is valid only in the limit of a magnetic field weak enough that Zeeman splitting is small compared to the local spectral line width (i.e., typically for fields of the order of a $\mathrm{kG}$ or less for optical metal spectral lines, or of order $10 \mathrm{kG}$ for Balmer lines); (ii) it applies to isolated, unblended lines; (iii) the value of $g_{\text {eff }}$ varies by a significant amount from line to line; the use of an average value for all lines means that the actual $\left\langle B_{z}\right\rangle$ value varies from the computed value in individual lines by up to $\sim 25 \%$.

Bagnulo et al. (2002) proposed that Eq. (1) could be applied to spectropolarimetric data obtained with FORS1, using a least-squares technique that was subsequently extensively adopted in many surveys. Although field measurements obtained with Eq. (1) cannot be used for detailed modelling, this technique is certainly valuable to decide whether a star is magnetic or not, and to estimate the field strength with a reasonable approximation.

\section{FORS1 ARCHIVE DATA REDUCTION}

All archive data obtained in fast mode have been pre-processed using the ESO FORS pipeline (Izzo et al. 2010, 2011). Although this software tool is capable of delivering the final Stokes profiles, we preferred to develop a few algorithms that further improve the quality of the results. Therefore, instead of adopting the final products of the pipeline (i.e., the Stokes profiles), we have used the individual fluxes corresponding to the parallel 
and perpendicular beams split by the Wollaston prism (obtained at the various positions of the retarder waveplate), and combined them as explained below.

We adopt the same formalism used in Bagnulo et al. (2009), i.e., $f^{\|}$and $f^{\perp}$ are the fluxes in the parallel and in the perpendicular beam of the polarization analyser, respectively, $P_{V}=V / I$ is the circular polarization normalised to the intensity, and $N_{V}$ is the null profile, a quantity that was introduced by Donati et al. (1997), and that is representative of the noise of $P_{V}$ (see Bagnulo et al. 2009). As a quality check, a magnetic field can be deduced from the null profiles using the same formulas adopted to measure $\left\langle B_{z}\right\rangle$. The diagnostic content of this null field value, $\left\langle N_{z}\right\rangle$, will be discussed in Sect. 3.2.

The extracted fluxes $f^{\|}$and $f^{\perp}$ are usually combined to obtain the $P_{V}$ and $N_{V}$ profiles using the formulas of the difference methods given in Eqs. (A2) and (A7) of Bagnulo et al. (2009), which for convenience we reproduce below:

$$
\begin{aligned}
P_{V} & =\frac{1}{2 N} \sum_{j=1}^{N}\left[\left(\frac{f^{\|}-f^{\perp}}{f^{\|}+f^{\perp}}\right)_{\alpha_{j}}-\left(\frac{f^{\|}-f^{\perp}}{f^{\|}+f^{\perp}}\right)_{\alpha_{j}+90^{\circ}}\right] \\
N_{V} & =\frac{1}{2 N} \sum_{j=1}^{N}(-1)^{(j-1)}\left[\left(\frac{f^{\|}-f^{\perp}}{f^{\|}+f^{\perp}}\right)_{\alpha_{j}}-\left(\frac{f^{\|}-f^{\perp}}{f^{\|}+f^{\perp}}\right)_{\alpha_{j}+90^{\circ}}\right],
\end{aligned}
$$

where $\alpha_{j}$ belongs to the set $\left\{-45^{\circ}, 135^{\circ}\right\}$. We note that instead of setting the $\lambda / 4$ retarder waveplate to all four possible angles, most of the observers preferred to set it only to the angles $-45^{\circ}$ and $+45^{\circ}$.

In practice, in many cases we found the $P_{V}$ profile slightly offset from zero, even when no circular polarization of the continuum was expected. A possible explanation is cross-talk from linear to circular polarization, as discussed by Bagnulo et al. (2009). However, we often found slight but noticeable offsets also in FORS data for stars that are not linearly polarized. These offsets can be naturally explained if the ratio between the transmission functions in the perpendicular beam, and the transmission function in the parallel beam, does not remain constant as the retarder waveplate is set to the different position angles. The ratio between the transmission functions can be estimated from the ratios of the $f^{\|}$and $f^{\perp}$ fluxes in each frame, and analytically propagated to the final expressions of the $P_{V}$ profiles using Eqs. (3) of Bagnulo et al. (2011).

Both $P_{V}$ and $N_{V}$ profiles show occasional spikes that occur in the same wavelength bin. Most of these spikes are probably produced by cosmic ray hits, and, if not removed, may lead to spurious detections of magnetic fields, or at least decrease the precision of its determination. As a remedy, we clip the $P_{V}$ profiles by discarding from the computation of the $\left\langle B_{z}\right\rangle$ values those points for which the (rectified) $N_{V}$ value departs from zero by more than $3 \sigma$.

Figure 1 shows the results obtained for the FORS observations of the well known magnetic star HD 94660.

\subsection{Errors bars}

The uncertainties of the $\left\langle B_{z}\right\rangle$ measurements can be calculated according to a well established error theory; assuming that each individual step of the measurement process 

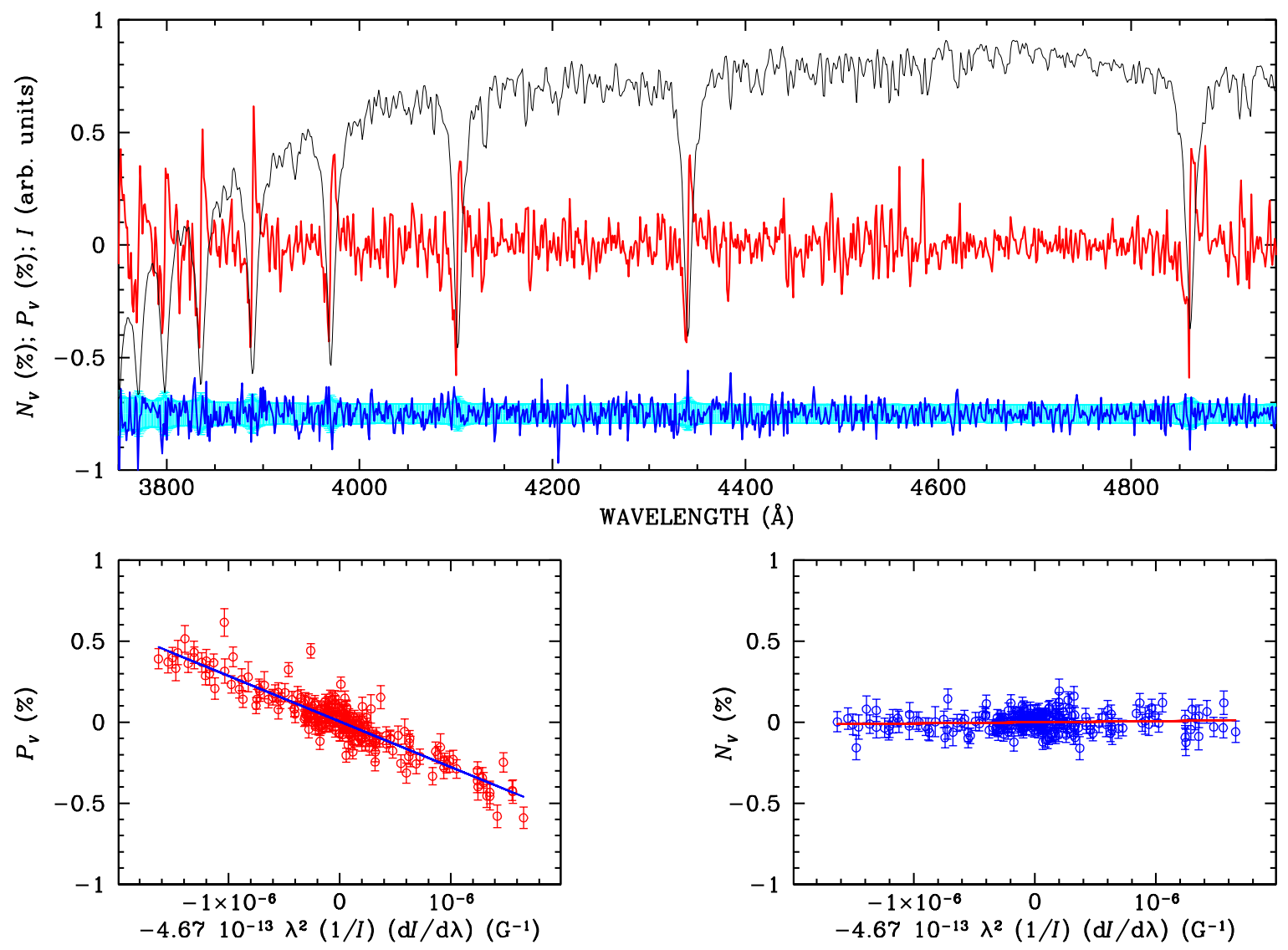

FIGURE 1. The polarized spectrum of the magnetic Ap star HD 94660. The top panel shows Stokes $I$ (black solid line, in arbitrary units, and not corrected for the instrument response), $P_{V}$ (red solid line centred about 0 ), and the null profile (blue solid line, offset by $-0.75 \%$ for display purpose). Light blue bars represent the error bars of $P_{V}$. The slope of the red interpolating lines in the bottom panels gives the mean longitudinal field from $P_{V}$ (left panel) and from the null profile (right panel, the latter being expected zero), both calculated using the H Balmer lines only. (After Bagnulo et al. 2011).

can be linearised, one can propagate the error bars of the measured flux to the $P_{V}$ profile (see, e.g., Eqs. (A3-A6) of Bagnulo et al. 2009), and then obtain the $\sigma_{\left\langle B_{z}\right\rangle}$ error bar associated to the $\left\langle B_{z}\right\rangle$ measurement. However, since there are indications that $\left\langle B_{z}\right\rangle$ error bars may be underestimated, we have preferred to adopt the more conservative approach to multiply the analytical expression for $\sigma_{\left\langle B_{z}\right\rangle}$ by the square root of the reduced $\chi^{2}$. The validity of this approach has been tested with Monte Carlo simulations. We have considered all raw frames associated to a certain number of measurements, and calculated the $\left\langle B_{z}\right\rangle$ and $\left\langle N_{z}\right\rangle$ values and their error bars. Then we have re-considered the same series of frames and scattered each pixel of each scientific frame according to a Gaussian distribution with $\sigma$ equal to the error pertaining to that pixel. We have re-reduced the so altered set of frames, and measured the $\left\langle B_{z}\right\rangle$ and $\left\langle N_{z}\right\rangle$ values from the $\mathrm{H}$ Balmer lines only and from the full spectrum. We have repeated this procedure 5000 times, and obtained for each set of frames two distributions of $\left\langle B_{z}\right\rangle$ values and two 

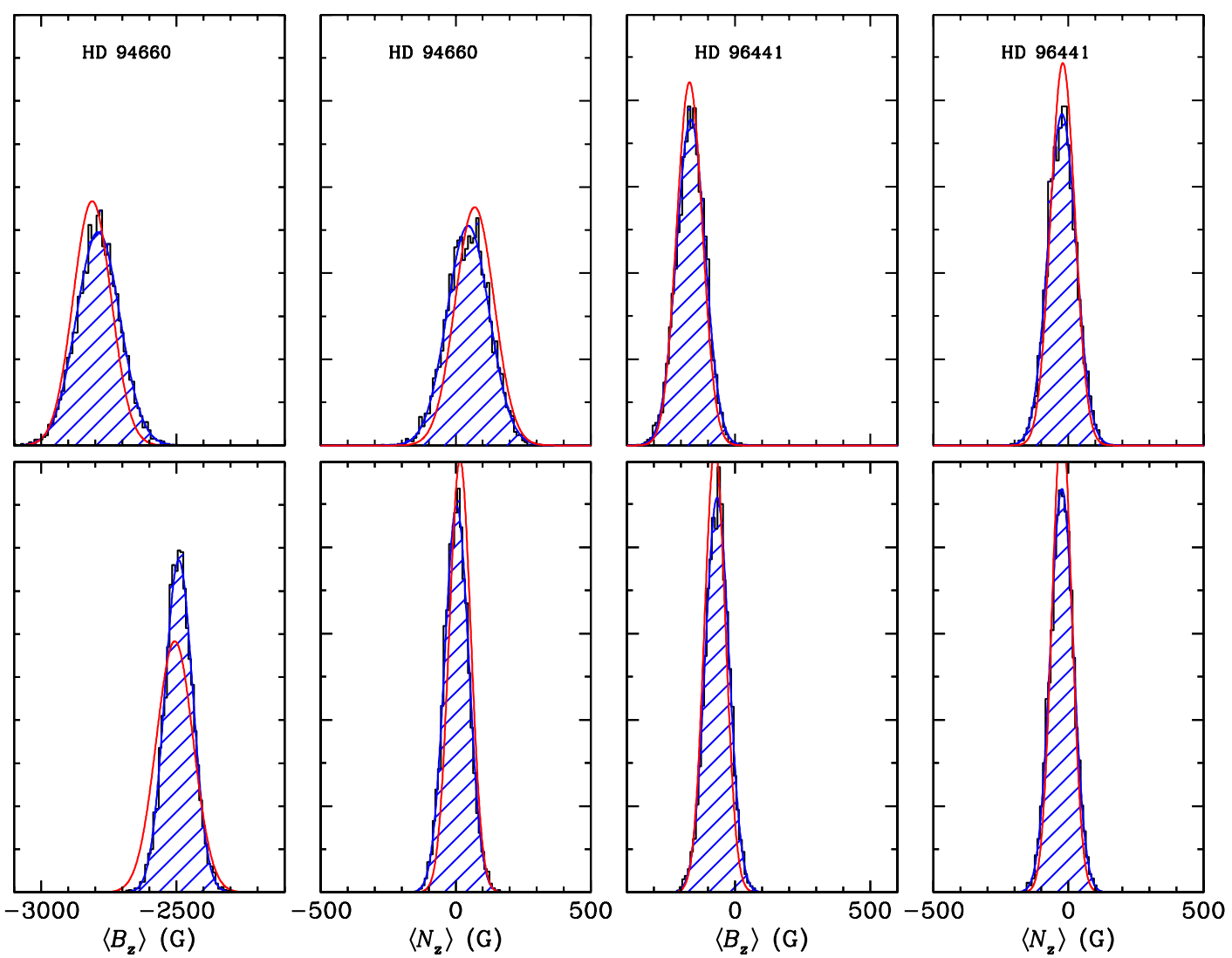

FIGURE 2. Monte Carlo simulations compared to predictions of the "standard" error theory for a strongly magnetic star (HD 94660) and a non magnetic star (HD 96441). Top panels refer to the $\left\langle B_{z}\right\rangle$ and $\left\langle N_{z}\right\rangle$ values obtained from the analysis of the $\mathrm{H}$ Balmer lines, and the bottom panels to the values obtained from the analysis of the full spectrum. Blue shaded histograms show the distributions of the $\left\langle B_{z}\right\rangle$ and $\left\langle N_{z}\right\rangle$ values obtained after Monte Carlo simulations. Red solid lines show Gaussian curves centred on the original $\left\langle B_{z}\right\rangle$ and $\left\langle N_{z}\right\rangle$ values with $\sigma$ calculated via usual standard error propagation.

distributions of $\left\langle N_{z}\right\rangle$ values. We have compared these distributions with the Gaussian curves centred on the $\left\langle B_{z}\right\rangle$ and $\left\langle N_{z}\right\rangle$ values measured from the unaltered frames, with $\sigma$ calculated via standard propagation theory. The results for two specific cases are shown in Fig. 2. The discrepancy observed for the case of $\left\langle B_{z}\right\rangle$ obtained from the analysis of the full spectrum of HD 96441 (left bottom panel of Fig. 2) may be due to the fact that our error bar calculated analytically is weighted by a reduced $\chi^{2}$. This value is definitely $>1$ because Eq. (1) is not strictly valid for blended metal lines when the field is strong. All the remaining cases shown in Fig. 2 confirm that $\left\langle B_{z}\right\rangle$ and $\left\langle N_{z}\right\rangle$ errors due to photon noise are correctly calculated. 


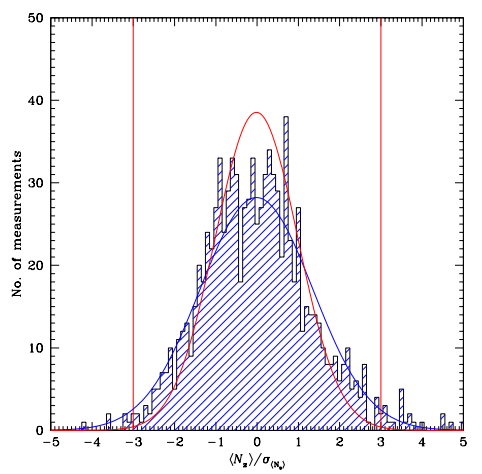

FIGURE 3. The actual distribution of the values $\left\langle N_{z}\right\rangle$ normalised to their error bars (shaded histogram) compared to their theoretical distribution, i.e., a Gaussian with $\sigma=1$. Vetical bars show the $\pm 3 \sigma$ limits. (After Bagnulo et al. 2011).

\subsection{Uncertainties not due to photon-noise}

There are indications that error bars due to photon-noise underestimate the actual uncertainties.

As mentioned in Sect. 1, in the literature there are non negligible discrepancies between the $\left\langle B_{z}\right\rangle$ values obtained by different authors who analyse the same FORS dataset. Note also that sometimes, the same authors have revised their own field determinations. This is probably due to the fact that $\left\langle B_{z}\right\rangle$ measurements depend, sometimes crucially, on the numerous specific choices taken during data reduction. For instance, the $\left\langle B_{z}\right\rangle$ and $\left\langle N_{z}\right\rangle$ values depend on the algorithm adopted for spectral extraction, on how data are clipped to remove the effects of cosmic rays, on whether flat-field correction is applied or not. A complete description of all these issues would be outside of the scope of anything but a detailed monograph, hence here we limit ourselves to refer the reader to a few numerical examples given in Bagnulo et al. (2011), and to warn that changes in the data reduction algorithm may lead to change of the $\left\langle B_{z}\right\rangle$ values comparable to $1 \sigma$.

A global inconsistency emerges from the analysis of the full FORS archive dataset, even when all data are reduced by consistently adopting the same algorithm, i.e., an unusually large number of field detections in the null profiles. Figure 3 shows the distribution of the null field values normalised to their error bars compared to the expected Gaussian with $\sigma=1$. This anomaly could be explained by a number of reasons, e.g., (i) that for some dataset, data reduction needs an extremely refined tuning; (ii) stars change radial velocity during an observing series; (iii) during the observations there are instrument flexures. It is likely that all these issues play a role, but we should note that in particular, instrument flexures, if present, would be completely out of user control. A flexure as small as a quarter of a pixel cannot be detected with the monitoring of the position of sky lines, because most of the observations were obtained with very short exposure times, and because in general the observed spectral range includes only one telluric line. Yet, simple numerical simulations show that even such small flexures may change the $\left\langle B_{z}\right\rangle$ and $\left\langle N_{z}\right\rangle$ values by an amount comparable to that of the error bars due to photon-noise. Occasional flexures of the order of 1 pixel could be responsible for the 
observed high frequency of $\left\langle N_{z}\right\rangle$ detections (about $3 \%-5 \%$ ).

\section{RESULTS}

The magnetic field measurements obtained with FORS1 have been directed at three main goals: detection of previously unknown fields, measurements of fields in stars already known to be magnetic, and establishing upper limits to fields in stars which are not magnetic.

Our discussion of reduction procedures for FORS1 spectropolarimetric data shows that using different reduction procedures to infer magnetic field strengths leads to different results. These include changes in both the value of the mean longitudinal field $\left\langle B_{z}\right\rangle$ and the associated uncertainty $\sigma_{\left\langle B_{z}\right\rangle}$ obtained from any specific observation.

This finding is probably not a serious concern for measurements of fields at the tens or hundreds of $\sigma$ level, as these measurements are much more sensitive to such factors as the lines available in the spectrum, the spectral region used, and the degree of chemical and inhomogeneity in the atmosphere.

On the other hand, underestimating the actual measurement uncertainty can have quite important effects when the issue is whether or not a field has been detected at all in a particular star or class of stars. Failure to recognize that computed uncertainties may not fully represent the range of measurement values that may be taken by $\left\langle B_{z}\right\rangle$ even in the absence of a real stellar field can lead to spurious discoveries. These in turn can lead to much effort being expended to analyse or explain the presence of fields that are not in fact there, or at least have not been reliably detected. It is in this context that the most harmful effects of incorrect measurements, or underestimated measurement uncertainties, can occur.

Because of problems that spurious discovery announcements can cause in a research field, we have carried out a systematic analysis of new magnetic field detections obtained at less than about the $6 \sigma$ level. In the following we report on some of our findings. Our complete revision of FORS1 magnetic data, which will include more classes of stars, will be published in two forthcoming papers (Bagnulo et al. 2011, and in preparation).

Herbig AeBe stars. The first measurement of a magnetic field in a Herbig Ae star obtained with the FORS1 instrument was reported by Hubrig et al. (2004), who measured $\left\langle B_{z}\right\rangle=-450 \pm 93 \mathrm{G}$ in HD 139614. This discovery was deemed as spurious detection by Wade et al. (2005) (who from the same FORS1 data measured $\left\langle B_{z}\right\rangle=$ $-150 \pm 50 \mathrm{G}$ ), but re-affirmed as real by Hubrig et al. (2006c). A new reduction by Hubrig et al. (2007a) led to the revised value of $\left\langle B_{z}\right\rangle=-112 \pm 36 \mathrm{G}$, and our rereduction of the discovery observation $\left(\left\langle B_{z}\right\rangle=-84 \pm 65 \mathrm{G}\right)$ confirms that no field is detected in this measurement. The same star was observed several times using the highresolution spectropolarimeter ESPaDOnS (Wade et al. 2005, 2007), and no magnetic field was ever detected.

The results of a FORS1 survey of 49 Herbig AeBe stars were described by Wade et al. $(2005 ; 2007)$. Magnetic fields were reported in two stars: HD 72106A (whose companion HD 72106B is a Herbig star), and HD 101412. The uncertainties of the measurement of these two stars have increased in our re-reduction of these data, but both 
fields are still detected at $3 \sigma$ level. These discoveries are corroborated by independent observations obtained with ESPaDOnS. Wade et al. $(2005 ; 2007)$ report also marginal detections in other three Herbig stars, but these detections were never confirmed by subsequent observations, nor by our new data reduction.

Further surveys of Herbig AeBe stars using FORS1 were described by Hubrig et al. (2006c; 2007a), who reported the discovery of magnetic fields in two new Herbig stars. Hubrig et al. (2009b) report new magnetic field detections in six more Herbig stars (almost $30 \%$ of the observed sample). In our re-reduction of all these data, only three measurements remain signficant. Detections in HD 144432 and in HD 144668 (at $3 \sigma$ level) might be real, but require confirmation. A detection at the $5-6 \sigma$ level of a field in HD 150193 appears stronger in our re-reduction than originally published. However, a single ESPaDOnS observation with an uncertainty of about $15 \mathrm{G}$ detects no field (E. Alecian, private communication). In conclusion, most of FORS1 field discoveries of Herbig AeBe stars appear to be spurious, and detectable magnetic fields appear to occur in only $\leq 10 \%$ of all Herbig AeBe stars observed with FORS1.

HgMn stars. Some of the peculiar A stars of the HgMn type, and in particular $\alpha$ And $=$ HD 358, have been repeatedly observed with FORS1. Based on these data, Hubrig et al. (2006b) have claimed field detections in four $\mathrm{HgMn}$ stars.

Numerous investigations of magnetism in HgMn (e.g., Borra \& Landstreet 1980, Shorlin et al. 2002, Makaganiuk et al. 2011) have found no convincing evidence for fields in this class of stars with uncertainties often in the range of a few $G$ up to a few tens of G. Furthermore, $\alpha$ And has been specifically investigated with the MuSiCoS (13 observations, typical $\sigma \sim 30-60 \mathrm{G}$ ) and ESPaDOnS (5 observations, typical $\sigma \sim$ $6-19$ G) spectropolarimeters without any significant detection Wade et al. (2006). Our new reductions have increased the standard errors of all FORS1 measurements by factors of 1.3 , and none of our results shows a field at the $3 \sigma$ level.

$\beta$ Cephei pulsators and Slowly Pulsating B stars. Hubrig et al. (2006a) and Hubrig et al. (2009a) have reported the discovery of a field in the $\beta$ Cep star $\xi^{1} \mathrm{CMa}=$ HD 46328, based on altogether 13 detections at approximately the 6 or $7 \sigma$ level. The field always appears to be close to $+350 \mathrm{G}$. This detection is supported by a single field detection by Silvester et al. (2009) using ESPaDOnS at about the $30 \sigma$ level. Our rereduction of these data also confirms the presence of a field. This discovery appears very robust, and clearly indicates the capability of FORS1 to detect rather modest magnetic fields.

Major FORS1 surveys of both $\beta$ Cep and SPB stars have been carried out by Hubrig et al. (2006a) and Hubrig et al. (2009a). Nearly 70 stars were observed for magnetic fields using the FORS1 spectropolarimeter (19 known and suspected $\beta$ Cep stars, 50 known and suspected SPBs). Fields were reported to have been detected in five $\beta$ Cep stars and 26 SPB stars. If these results were confirmed they would be quite important. Such high incidence of detected fields would strongly suggest that magnetism is intrinsically connected with the pulsation phenomenon in early B stars, as it is in the cool rapidly oscillating Ap (roAp) stars.

However, all reported field detections in $\beta$ Cep stars, except those of $\xi^{1} \mathrm{CMa}$, have mostly become insignificant in the new reductions. Only two field measurements, one 
each for HD 74575 and HD 136504, are still barely significant at the $3 \sigma$ level. Of the more than 40 field detections reported in SPB stars by Hubrig et al. (2006a) and Hubrig et al. (2009a), all but five have decreased to non-detections in the new reductions. Our reduction suggest that fields might be present in HD 53921, HD 152511 (with two still apparently significant detections), and HD 208057. However, some or all of these detections could be manifestations of the occasional outliers found in the FORS1 data. Note that a field has also been marginally detected by Silvester et al. (2009) in a single ESPaDOnS measurement of HD 208057.

Based on the re-reductions of the FORS1 data as well as other published material, we conclude that as in other kinds of upper main sequence stars, magnetic fields are relatively rare in $\beta$ Cep and SPB stars. Furthermore, there is no significant case for considering that the pulsation properties of these B pulsators are intrinsically connected to the presence of weak magnetic fields.

O-type stars. Hubrig et al. (2008) carried out the first large survey of O-type stars with FORS1, and reported field measurements of 13 stars (including one star previously reported by Hubrig et al. 2007b). Field discoveries were claimed for five of these stars. Our reduction confirms a marginal detection in only one of these stars, the O6.5f?p star HD 148937. The field of this star has been detected in three more observations with FORS2 by Hubrig et al. (2011) and with ESPaDONs observations obtained by Wade et al. (2011). This discovery is clearly real, although the field of this star, always about $-250 \mathrm{G}$, is close to the limit for reliable field detection by FORS1 for such stars.

Central stars of planetary nebulae. Jordan et al. (2005) carried out six field measurements of four central stars of planetary nebulae (CSPN), and concluded that at least two of these stars have highly significant fields in the $\mathrm{kG}$ range. This result supported the idea that such fields have an important shaping effect on the the planetary nebulae themselves, and suggest that there must have been important loss of magnetic flux between this evolution state and the white dwarf state where stars with as much flux as that inferred for the CSPN are relatively rare. However, this result was called into question recently by Leone et al. (2011), who obtained one new measurement of each of the CSPNs NGC 1360 and LSS 1362, detecting no significant field in either star. Leone et al. (2011) also re-reduced the older data of Jordan et al. (2005), finding no significant fields. Our new reductions are completely consistent with the re-reductions carried out by Leone et al. (2011). None of the original field measurements of Jordan et al. (2005) reveals a significant field. As remarked by Leone et al. (2011), there is now no significant evidence for coherent magnetic fields in the central stars of planetary nebulae, and the current best upper limits on $\left\langle B_{z}\right\rangle$ are at roughly the 1 to $2 \mathrm{kG}$ level.

\section{CONCLUSIONS}

FORS is an instrument perfectly capable of performing field measurements of the order of at least $250-300 \mathrm{G}$ even in faint and rapidly rotating stars. However, for weaker fields, it is well possible that limits intrinsic to the technique, or even to the instrument itself, are reached. In particular, we have shown that (i) different data reduction techniques lead 
to results that differ for values comparable to (and in some cases larger than) photonnoise error bars; (ii) instrument flexures as small as a quarter of a pixel may affect the field measurements by an amount again comparable to a typical photon-noise error bar. Since FORS is mounted at the Cassegrain focus, such small flexures might occasionally occur. We conclude that, before being accepted as real, many recent claims of weak field detections obtained with FORS in various kinds of stars need to be confirmed by new and repeated measurements obtained both with FORS itself and, independently, with other instruments.

\section{ACKNOWLEDGMENTS}

Carlo Izzo was responsible for the pipelines of various instruments of the La SillaParanal Observatory, including the FORS instrument. Without his help and friendship this work would not have been possible. Carlo died after short illness a few days before this workshop. He will be deeply missed by many friends inside and outside ESO.

\section{REFERENCES}

1. Appenzeller, I., Fricke, K., Furtig, W., et al. 1998, The Messenger, 94, 1

2. Bagnulo, S., Szeifert, T., Wade, G.A., Landstreet, J.D., \& Mathys, G. 2002, A\&A, 389, 191

3. Bagnulo, S., Landolfi, M., Landstreet, J.D. et al. 2009, PASP, 121, 993

4. Bagnulo, S., Landstreet, J.D., Fossati, L., \& Kochukhov, O. 2011, A\&A (submitted)

5. Borra, E.F., \& Landstreet, J.D. 1980, ApJS, 42, 421

6. Donati, J.-F., Semel, M., Carter, B.D., et al. 1997, MNRAS, 291, 658

7. Izzo, C., de Bilbao, L., Larsen, J. et al. 2010, SPIE, 7737, 773729

8. Izzo, C., de Bilbao, L., Larsen, J.M. 2011 FORS Pipeline User Manual, Issue 4.1, VLT-MANESO_19500-4106

9. Jordan, S., Werner, K., \& O’Toole, S.J. 2005, A\&A, 432, 273

10. Hubrig, S., Schöller, M., \& Yudin, R.V. 2004, A\&A, 428L, 1

11. Hubrig, S., Briquet, M., Schöller, M. et al. 2006a, MNRAS, 369, 61

12. Hubrig, S., North, P., Schöller, M., \& Mathys, G. 2006b, AN, 327, 289

13. Hubrig, S., Yudin, R.V., Schöller, M., Pogodin, M.A. 2006c, A\&A, 446, 1089

14. Hubrig, S., Pogodin, M.A., Yudin, R.V., Schöller, M., \& Schnerr, R.S. 2007a, A\&A, 463, 1039

15. Hubrig, S., Yudin, R.V., Pogodin, M., Schöller, M., \& Peters, G.J. 2007b, AN, 328, 1133

16. Hubrig, S., Schöller, M., Schnerr, R.S. et al. 2008, A\&A, 490, 793

17. Hubrig, S., Briquet, M., De Cat, P. et al. 2009a, AN, 330, 317

18. Hubrig, S., Stelzer, B., Schöller, M., et al. 2009b, A\&A, 502, 283

19. Hubrig, S., Mikulášek, Z., González, J. F. et al. 2011 A\&A, 525, L4

20. Leone, F., Martínez González, M.J., Corradi, R.L.M., Privitera, G., Mason Sainz, R. 2011, ApJ, 731L 33

21. Makaganiuk, V., Kochukhov, O., Piskunov, N., et al. 2011, A\&A, 529, A160

22. Shorlin, S. L. S., Wade, G.A., Donati, J.-F. et al. 2002, A\&A, 392, 637

23. Silvester, J., Neiner, C., Henrichs, H.F., et al. 2009 MNRAS, 398, 1505

24. Wade, G.A., Drouin, D., Bagnulo, S., et al. 2005, A\&A, 442, 31L

25. Wade, G.A., Aurière, M., Bagnulo, S., et al. 2006, A\&A, 451, 293

26. Wade, G.A., Bagnulo, S., Drouin, D., Landstreet, J.D., \& Monin, D. 2007, MNRAS, 376, 1145

27. Wade, G. A., Grunhut, J., Gräfener, G. et al. 2011, A\&A, in press (arXiv:1108.4847) 